\title{
On geometric quantization of the Dirac magnetic monopole
}

Graham M. Kemp, Alexander P. Veselov

To cite this article: Graham M. Kemp, Alexander P. Veselov (2014) On geometric quantization of the Dirac magnetic monopole, Journal of Nonlinear Mathematical Physics 21:1, 34-42, DOI: https://doi.org/10.1080/14029251.2014.894719

To link to this article: https://doi.org/10.1080/14029251.2014.894719

Published online: 04 January 2021 


\title{
On geometric quantization of the Dirac magnetic monopole
}

\author{
Graham M. Kemp \\ Department of Mathematical Sciences, Loughborough University, \\ Loughborough, Leicestershire, LE11 3TU, UK \\ G.Kemp@lboro.ac.uk \\ Alexander P. Veselov \\ Department of Mathematical Sciences, Loughborough University, \\ Loughborough, Leicestershire, LE11 3TU, UK \\ Moscow State University, Moscow 119899, Russia \\ A.P.Veselov@lboro.ac.uk
}

Received 1 October 2013

Accepted 19 November 2013

\begin{abstract}
We give a simple derivation of the spectrum of the Dirac magnetic monopole on a unit sphere $S^{2}$ based on geometric quantization and the Frobenius reciprocity formula. The starting point is the calculation by Novikov and Schmelzer of the canonical symplectic structure on the coadjoint orbits of the isometry group of 3-dimensional Euclidean space $E(3)$, which showed the appearance of the Dirac magnetic term.
\end{abstract}

Keywords: Dirac magnetic monopole; geometric quantization; Frobenius reciprocity formula.

2000 Mathematics Subject Classification: 53D50, 81R12

\section{Introduction}

The Dirac magnetic monopole is one of the most remarkable and one of the first integrable systems of quantum mechanics. In his pioneering paper [4] Dirac showed that an isolated magnetic charge $q$ should be quantized: $q \in \mathbb{Z}$. The corresponding Schrödinger equation was solved by Tamm [18] while he was visiting Dirac in Cambridge in 1931.

On the other hand it took quite a while to understand the global nature of the corresponding eigenfunctions. For the Dirac magnetic monopole on a sphere it was done only in 1976 by Wu and Yang [20], who explained that the corresponding wavefunctions (known as monopole harmonics) are sections of a complex line bundle $L$ over $S^{2}$ and found the spectrum to be

$$
\lambda=\left[l(l+1)+|q|\left(l+\frac{1}{2}\right)\right], l=0,1,2, \ldots \text { with degeneracy } 2 l+|q|+1 .
$$

This gives also a geometric interpretation of Dirac's quantization condition: magnetic charge is the first Chern class $c_{1}(L)=q$ of the bundle $L$, which must be an integer.

A different derivation of this result was given by Ferapontov and one of the authors [6], who extended the classical factorisation method going back to Darboux and Schrödinger [17] to curved surfaces. This provided an explicit description of the monopole harmonics by recursive application of the lowering operators to the ground states, which, under the isomorphism $S^{2} \cong \mathbb{C} P^{1}$, are given for positive $q$ by polynomials of degree $\leq q$. 
We present here a simple derivation of the spectrum of the Dirac monopole on a unit sphere using geometric quantization. We should say that geometric quantization of the Dirac magnetic monopole and related problems were already discussed in [14,19], but we believe that our approach is simpler and clearer.

The initial point for us was the calculation by Novikov and Schmelzer [15] of the canonical symplectic structure on the coadjoint orbits of the Euclidean group $E(3)$ of motions of $\mathbb{E}^{3}$, which showed the relation with the classical Dirac monopole (see the next section). A similar calculation for Poincare and Galilean groups was done by Reiman [16], who also seems to have the idea of geometric quantization in mind, but did not pursue it.

We first show that Novikov-Schmelzer variables have a natural quantum version as covariant derivatives acting on the space of sections $\Gamma(L)$ of the corresponding line bundle $L$. Fierz's modification [7] of the angular momentum in the presence of the Dirac magnetic monopole appears naturally in this relation.

The space $\Gamma(L)$ is the representation of $S U(2)$ induced from the representation of $U(1) \subset S U(2)$ given by $z \rightarrow z^{q}, z \in U(1)$. This space can be decomposed as an $S U(2)$-module using the classical Frobenius reciprocity formula [8]. We show that the formula for the Dirac monopole spectrum (1.1) is a simple corollary of this.

In the last section we discuss the generalisations of this to any coadjoint orbit of a compact Lie group.

\section{The coadjoint orbits of the Euclidean group $E(3)$}

Let $e(3)$ be the Lie algebra of the Euclidean group $E(3)$ of motions of $\mathbb{E}^{3}$. It has the basis $l_{1}, l_{2}, l_{3}, p_{1}, p_{2}, p_{3}$, where $p$ and $l$ are generators of translations and rotations (momentum and angular momentum) respectively.

The dual space $e(3)^{*}$ with the coordinates $\left\{l_{1}, l_{2}, l_{3}, p_{1}, p_{2}, p_{3}\right\}$ has the canonical Poisson bracket

$$
\left\{l_{i}, l_{j}\right\}=\varepsilon_{i j k} l_{k}, \quad\left\{l_{i}, p_{j}\right\}=\varepsilon_{i j k} p_{k}, \quad\left\{p_{i}, p_{j}\right\}=0 .
$$

Its symplectic leaves are the coadjoint orbits of $E(3)$, which are the level sets of the Casimir functions

$$
C_{1}:=(p, p)=R^{2}, \quad C_{2}:=(l, p)=\alpha R
$$

Following Novikov and Schmelzer [15] introduce

$$
\sigma_{i}=l_{i}-\frac{\alpha}{R} p_{i}
$$

to identify the coadjoint orbits with $T^{*} S^{2}$ :

$$
(p, p)=R^{2}, \quad(\sigma, p)=0
$$

where $T S^{2}$ and $T^{*} S^{2}$ have been identified using the standard Riemannian metric on the radius $R$ sphere. 
The new coordinates $\left\{\sigma_{1}, \sigma_{2}, \sigma_{3}, p_{1}, p_{2}, p_{3}\right\}$ have Poisson brackets

$$
\left\{\sigma_{i}, \sigma_{j}\right\}=\varepsilon_{i j k}\left(\sigma_{k}-\frac{\alpha}{R} p_{k}\right),\left\{\sigma_{i}, p_{j}\right\}=\varepsilon_{i j k} p_{k},\left\{p_{i}, p_{j}\right\}=0
$$

Novikov and Schmelzer computed the canonical symplectic form on the coadjoint orbits and showed that it is given by

$$
\omega=\mathrm{d} P \wedge \mathrm{d} Q+\frac{\alpha}{R^{2}} \mathrm{~d} S
$$

where $\mathrm{d} P \wedge \mathrm{d} Q$ is the standard symplectic form on $T^{*} S^{2}$ and $\mathrm{d} S$ is the area form on $S^{2}$ (see also [16]). As it was pointed out in [15] the second term corresponds to the magnetic field of the (nonquantized) Dirac monopole:

$$
\mathscr{H}=\frac{\alpha}{R^{2}} \mathrm{~d} S
$$

The value

$$
q=\frac{1}{2 \pi} \int_{S^{2}} \mathscr{H}
$$

is called the charge of the Dirac monopole. Dirac's quantization condition [4] is

$$
q=\frac{1}{2 \pi} \int_{S^{2}} \mathscr{H}=\frac{1}{2 \pi} \int_{S^{2}} \frac{\alpha}{R^{2}} \mathrm{~d} S=2 \alpha \in \mathbf{Z} .
$$

Comparing this with (2.3) we see that this is identical to the geometric quantization condition [11]:

$$
\frac{1}{2 \pi} \int_{\left[S^{2}\right]} \omega=\frac{1}{2 \pi} \int_{S^{2}} \frac{\alpha}{R^{2}} \mathrm{~d} S \in \mathbf{Z}
$$

\section{Line bundles over $S^{2}$ and quantization of Novikov-Schmelzer variables}

It is convenient to use the scaled variables

$$
x_{i}=p_{i} / R, \quad x^{2}=x_{1}^{2}+x_{2}^{2}+x_{3}^{2}=1
$$

to work with the unit sphere $S^{2}$.

The quantum version of the Poisson brackets (2.2) are the following commutation relations (we are using the units in which Planck's constant $\hbar=1$ )

$$
\left[\hat{\sigma}_{k}, \hat{\sigma}_{l}\right]=i \varepsilon_{k l m}\left(\hat{\sigma}_{m}-\alpha \hat{x}_{m}\right),\left[\hat{\sigma}_{k}, \hat{x}_{l}\right]=i \varepsilon_{k l m} \hat{x}_{m},\left[\hat{x}_{k}, \hat{x}_{l}\right]=0 .
$$

We are going to show now that this algebra has a natural representation on the space of sections of a certain line bundle over $S^{2}$.

Recall that a connection on a vector bundle $\xi$ over manifold $M^{n}$ associates to every vector field $X$ on $M^{n}$ the operator of covariant derivative $\nabla_{X}$ acting on sections of $\xi$. The corresponding curvature tensor $\mathscr{R}$ is defined for each pair of vector fields $X, Y$ as

$$
\mathscr{R}(X, Y)=\nabla_{X} \nabla_{Y}-\nabla_{Y} \nabla_{X}-\nabla_{[X, Y]},
$$

where $[X, Y]$ is the standard Lie bracket of vector fields (see e.g. [13]). 
Consider a complex line bundle over $S^{2}$ with a $U(1)$-connection having the curvature form

$$
\mathscr{R}=i \mathscr{H}=i \alpha \mathrm{d} S
$$

which is motivated by geometric quantization. Since the first Chern class of the bundle must be an integer we have

$$
q=\frac{1}{2 \pi i} \int_{S^{2}} \mathscr{R}=\frac{1}{2 \pi} \int_{S^{2}} \alpha \mathrm{d} S=2 \alpha \in \mathbf{Z},
$$

which is precisely Dirac's quantization condition.

Let

$$
X_{1}=x_{3} \partial_{2}-x_{2} \partial_{3}, X_{2}=x_{1} \partial_{3}-x_{3} \partial_{1}, X_{3}=x_{2} \partial_{1}-x_{1} \partial_{2}
$$

be the vector fields generating rotations of $S^{2}$ and $\nabla_{X_{j}}$ be the corresponding covariant derivatives. We claim that

$$
\hat{\nabla}_{j}:=i \nabla_{X_{j}}
$$

and the operators $\hat{x}_{j}$ of multiplication by $x_{j}$ satisfy the commutation relations (3.2).

Indeed, by definition of the curvature form, we have

$$
\mathscr{R}\left(X_{1}, X_{2}\right)=\nabla_{X_{1}} \nabla_{X_{2}}-\nabla_{X_{2}} \nabla_{X_{1}}-\nabla_{\left[X_{1}, X_{2}\right]}=i \alpha x_{3}
$$

since

$$
\alpha \mathrm{d} S\left(X_{1}, X_{2}\right)=\alpha\left|\begin{array}{ccc}
x_{1} & x_{2} & x_{3} \\
0 & x_{3} & -x_{2} \\
-x_{3} & 0 & x_{1}
\end{array}\right|=\alpha x_{3}\left(x_{1}^{2}+x_{2}^{2}+x_{3}^{2}\right)=\alpha x_{3}
$$

This implies

$$
\left[\nabla_{X_{1}}, \nabla_{X_{2}}\right]=\nabla_{X_{3}}+i \alpha \hat{x}_{3}
$$

since $\left[X_{1}, X_{2}\right]=X_{3}$. Similarly we have

$$
\left[\hat{\nabla}_{k}, \hat{\nabla}_{l}\right]=i \varepsilon_{k l m}\left(\hat{\nabla}_{m}-\alpha \hat{x}_{m}\right)
$$

for all $k, l, m=1,2,3$. The rest of the relations (3.2) are obvious.

Alternatively, we can look for the quantization of Novikov-Schmelzer variables as covariant derivatives:

$$
\hat{\sigma}_{j}=i \nabla_{X_{j}}
$$

Then the same calculation shows that the curvature form of the corresponding connection must be $i \alpha \mathrm{d} S$. 
Finally returning to the original variables we have the operators

$$
\hat{l}_{j}=\hat{\nabla}_{j}+\alpha x_{j}
$$

which coincides with the famous modification of the angular momentum in the presence of the Dirac magnetic monopole [7]. This provides us with one more explanation of this well known, but a bit mysterious ${ }^{a}$ physical notion.

\section{Induced representations and Frobenius reciprocity}

Let $L_{q}$ be the complex line bundle over $S^{2}$ with first Chern class $q$. We are interested in the space $\Gamma\left(L_{q}\right)$ of sections of $L_{q}$. Viewing $S^{2}$ as $S U(2) / U(1)$ (with $U(1)$ as the diagonal subgroup) we have a natural interpretation of $\Gamma\left(L_{q}\right)$ as a representation of $S U(2)$.

In representation theory this construction is known as an induced representation (see e.g. [8]). One can use the classical Frobenius reciprocity formula from this theory to decompose $\Gamma\left(L_{q}\right)$ into irreducible $S U(2)$ modules.

First recall that all finite-dimensional irreducible representations of $S U(2)$ are labelled by a highest weight $k \in \mathbf{Z}_{\geq 0}$. The corresponding spaces $V_{k}$ have dimension $k+1$ and weights

$$
-k,-k+2, \ldots, k-2, k
$$

All finite-dimensional irreducible representations $W_{q}$ of $U(1)$ have dimension 1 and are given by

$$
e^{i \theta} \mapsto e^{i q \theta}, q \in \mathbf{Z}
$$

One can use $W_{q}$ to induce a representation $\operatorname{ind}_{U(1)}^{S U(2)}\left(W_{q}\right)$ of $S U(2)$, which can be described geometrically as the space of sections of the line bundle $L_{q}$ over $S^{2}$ with the first Chern class $q$ (see e.g. [2]):

$$
\Gamma\left(L_{q}\right)=\operatorname{ind}_{U(1)}^{S U(2)}\left(W_{q}\right)
$$

This induced representation is not irreducible. To decompose it we will use the Frobenius reciprocity formula

$$
\left\langle V, \operatorname{ind}_{H}^{G}(W)\right\rangle_{G}=\left\langle W, \operatorname{res}_{G}^{H}(V)\right\rangle_{H}
$$

Here $G$ is a group, $H$ is its subgroup, $V$ and $W$ are the irreducible representations of $G$ and $H$ respectively, $\operatorname{ind}_{H}^{G}(W)$ is the representation of $G$ induced from $W, \operatorname{res}_{G}^{H}(V)$ is the restriction of the representation $V$ to the subgroup $H$ and the brackets denote the multiplicity of the first representation entering into the second one (see e.g. [8]).

\footnotetext{
${ }^{a}$ Sidney Coleman, in his famous lectures on Dirac monopoles [3], wrote about this modification of angular momentum:"The second term looks very strange indeed; in Rabi's immortal words about something else altogether,"Who ordered that?"'
} 
In our concrete case we have

$$
\left\langle V_{k}, \operatorname{ind}_{U(1)}^{S U(2)}\left(W_{q}\right)\right\rangle_{S U(2)}=\left\langle W_{q}, \operatorname{res}_{S U(2)}^{U(1)}\left(V_{k}\right)\right\rangle_{U(1)} .
$$

Since the restriction is the sum of the weight spaces

$$
\operatorname{res}_{S U(2)}^{U(1)}\left(V_{k}\right)=\bigoplus_{j \in S_{k}} W_{j}
$$

where $S_{k}=\{-k,-k+2, \ldots, k-2, k\}$ we see that each $V_{k}$, which (after restriction) contains $W_{q}$ will appear once in the decomposition of $\Gamma\left(L_{q}\right)$ and this can happen only if $k \geq|q|$ and $k-|q|$ is even. Therefore $\Gamma\left(L_{q}\right)$ decomposes into $S U(2)$-modules as

$$
\operatorname{ind}_{U(1)}^{S U(2)}\left(W_{q}\right)=\Gamma\left(L_{q}\right)=\bigoplus_{l \in \mathbb{Z}_{\geq 0}} V_{2 l+|q|} \cdot
$$

\section{Calculation of the spectrum of the Dirac monopole}

The Hamiltonian of the Dirac monopole can be written in terms of Novikov-Schmelzer operators as

$$
H=\hat{\sigma}^{2}
$$

or, equivalently, in terms of magnetic angular momentum $\hat{l}$ as

$$
H=\hat{l}^{2}-\alpha^{2}=\hat{l}^{2}-\frac{1}{4} q^{2}
$$

Since the components of $\hat{l}_{m}$ satisfy the standard commutation relations

$$
\left[\hat{l}_{k}, \hat{l}_{m}\right]=i \varepsilon_{k m n} \hat{l}_{n},
$$

the operator $\hat{l}^{2}$ is a Casimir operator for $S U(2)$ and acts on $V_{k}$ as a scalar: if $s=k / 2$ then

$$
\hat{l}^{2}=s(s+1)=\frac{1}{4} k(k+2)
$$

see e.g. [8]. The space $V_{2 l+|q|}$ has dimension $2 l+|q|+1$, and for $\psi \in V_{2 l+|q|}$, the operator $H$ acts as

$$
H \psi=\left(\hat{l}^{2}-\frac{1}{4} q^{2}\right) \psi=\left[\frac{1}{4}(2 l+|q|)(2 l+|q|+2)-\frac{1}{4} q^{2}\right] \psi=\left[l(l+1)+|q|\left(l+\frac{1}{2}\right)\right] \psi
$$

Thus for a Dirac monopole of charge $q$ the spectrum is

$$
\left[l(l+1)+|q|\left(l+\frac{1}{2}\right)\right], l \in \mathbb{Z}_{\geq 0} \text { with degeneracy } 2 l+|q|+1
$$

agreeing exactly with (1.1).

The corresponding eigenfunctions were computed using Darboux-Schrödinger factorisation method in [6], where the ground eigenstates were identified for non-negative $q$ with the space of polynomials of degree $\leq q$. In our picture the ground eigenspace corresponds to the subspace of holomorphic sections of Lq, which by the Borel-Weil theorem $[2,8]$ can be identified with the corresponding irreducible $\mathrm{SU}(2)$-module $V_{q}$. 


\section{Generalisation: Dirac magnetic monopole on coadjoint orbits}

We discuss briefly here the generalisation of this to any coadjoint orbit of a compact Lie group ${ }^{b}$ referring for the details to [10]. The classical case was studied by Efimov [5] and Bolsinov and Jovanovic [1].

Let $G$ be a Lie group, $\mathfrak{g}$ be its Lie algebra, $\mathfrak{g}^{*}$ be its dual space, $A d$ and $A d^{*}$ be the corresponding adjoint and coadjoint actions of $G$ on $\mathfrak{g}$ and $\mathfrak{g}^{*}$ :

$$
\begin{gathered}
A d_{g}(Y)=\left.\frac{d}{d s}\right|_{s=0} g \exp (s Y) g^{-1}=g Y g^{-1} . \\
\left\langle A d_{g}^{*}(f), X\right\rangle:=\left\langle f, A d_{g^{-1}} X\right\rangle .
\end{gathered}
$$

where $\langle f, X\rangle$ is the canonical pairing between $f \in \mathfrak{g}^{*}$ and $X \in \mathfrak{g}$. Similarly the adjoint and coadjoint actions $a d^{*}$ of $\mathfrak{g}$ on $\mathfrak{g}^{*}$ are defined by

$$
\begin{gathered}
a d_{X}(Y)=\left.\frac{d}{d t}\right|_{t=0} \exp (t X) Y \exp (-t X)=[X, Y], \\
\left\langle a d_{Y}^{*}(f), X\right\rangle:=-\left\langle f, a d_{Y} X\right\rangle=\langle f,[X, Y]\rangle .
\end{gathered}
$$

Given $a$ in $\mathfrak{g}^{*}$, the coadjoint orbit $\mathscr{O}(a)$ is defined by

$$
\mathscr{O}(a):=\left\{x \in \mathfrak{g}^{*}: x=A d_{g}^{*}(a), g \in G\right\}=A d_{G}^{*}(a) .
$$

Defining $G_{a}$ to be the stabilizer of the point $a, G_{a}:=\left\{g \in G: A d_{g}^{*}(a)=a\right\}$, it is clear that if $x=$ $A d_{g}^{*}(a)$ then $G_{x}=g\left(G_{a}\right) g^{-1}$. In this way, one may identify $\mathscr{O}(a)$ with the homogeneous space $\mathscr{O}(a) \cong G / G_{a}$.

Assume now that $G$ is compact, connected, simply-connected semi-simple Lie group. Such a group has a positive $A d$-invariant Cartan-Killing form (, ) defined on its Lie algebra $\mathfrak{g}$ (see e.g. [8]), which can be used to identify $\mathfrak{g}^{*}$ with $\mathfrak{g}$.

There are two natural metrics on $\mathscr{O}(a)$ : the metric induced from $\mathfrak{g}^{*}$ with Cartan-Killing form and the following normal metric defined by

$$
\left(\xi_{x}, \eta_{x}\right)_{n o r}:=(\pi(\xi), \pi(\eta))
$$

where $\pi$ is the projection map $\pi: \mathfrak{g} \rightarrow \mathfrak{g} / \mathfrak{g}_{x} \cong \mathfrak{g}_{x}^{\perp}$. We will be using the latter one.

Consider the following class of closed $G$-invariant 2-forms on $\mathscr{O}(a)$ (cf. [1]). Let $G_{x} \subset G$ be the stationary subgroup of $x \in \mathscr{O}(a)$ and $Z\left(\mathfrak{g}_{x}\right)$ be the centre of its Lie algebra. Choose $f_{a} \in\left(Z\left(\mathfrak{g}_{a}\right)\right)^{*}$ and define $f_{x}=A d_{g}^{*}\left(f_{a}\right) \in\left(Z\left(\mathfrak{g}_{x}\right)\right)^{*}$ at any other point $x=A d_{g}^{*}(x) \in \mathscr{O}(a)$.

\footnotetext{
${ }^{\mathrm{b}}$ We are very grateful to Alexey Bolsinov for illuminating discussions of this generalisation.
} 
Let $\xi_{x}=a d_{\xi}^{*}(x)$ and $\eta_{x}=a d_{\eta}^{*}(x)$ be two tangent vectors at $x$. Define a 2 -form on $T_{x} \mathscr{O}(a)$ by

$$
\sigma_{f}\left(\xi_{x}, \eta_{x}\right)=\left\langle f_{x},[\xi, \eta]\right\rangle .
$$

Then $\sigma_{f}$ defines an invariant, closed 2-form on $\mathscr{O}(a)$. When $f_{a}=a$ we have a canonical, $G$-invariant Kostant-Kirillov symplectic form

$$
\omega_{K K}\left(\xi_{x}, \eta_{x}\right)=\langle x,[\xi, \eta]\rangle .
$$

The forms $\sigma_{f}$ determine the class of $G$ invariant magnetic fields on $\mathscr{O}(a)$, which can be considered as classical analogues of Dirac magnetic monopoles. The original Dirac magnetic monopole discussed above corresponds to the simplest case $G=S O(3)$ and $\mathscr{O}(a)=S^{2}$. The Lie group $E(3)$ above in general case should be replaced by the semi-direct product $E G=G \ltimes_{A d} \mathfrak{g}$ with the product

$$
\left(g_{1}, X_{1}\right) \cdot\left(g_{2}, X_{2}\right)=\left(g_{1} g_{2}, A d_{g_{2}^{-1}} X_{1}+X_{2}\right) .
$$

The Lie algebra structure on $\mathfrak{e} \mathfrak{g}=\mathfrak{g} \ltimes_{A d} \mathfrak{g}$ is given by

$$
\left[\left(u_{1}, v_{1}\right),\left(u_{2}, v_{2}\right)\right]=\left(\left[u_{1}, u_{2}\right],\left[u_{1}, v_{2}\right]-\left[u_{2}, v_{1}\right]\right) .
$$

One can show [10] that the coadjoint orbit $\mathscr{O}(f, a)$ of a point $(f, a) \in \mathfrak{e g}^{*}$ with canonical KostantKirillov symplectic form is symplectomorphic to the magnetic cotangent bundle $\left(T^{*} \mathscr{O}(a), d p \wedge\right.$ $\left.d q+\pi^{*}\left(\omega_{f}\right)\right)$, which is a generalisation of Novikov-Schmelzer result.

By the generalised Dirac magnetic monopole on coadjoint orbit we mean the system on $T^{*} \mathscr{O}(a)$ with the symplectic form $\left.\omega=d p \wedge d q+\pi^{*}\left(\omega_{f}\right)\right)$ and the Hamiltonian given by the normal metric on $\mathscr{O}(a)$. Efimov [5] and Bolsinov and Jovanovic [1] proved the classical integrability in the case when the magnetic field is a multiple of Kirillov-Kostant form: $f=\alpha x$.

The quantisation gives the integrality condition for the corresponding form $\omega_{f}$ :

$$
\frac{1}{2 \pi} \int_{\sigma} \omega_{f} \in \mathbb{Z}
$$

for all $\sigma \in H_{2}(\mathscr{O}(a), \mathbb{Z})$. Such forms are in one-to-one correspondence with the characters of $H=G_{a}$ and with homogeneouss line bundles over $\mathscr{O}(a)=G / H$.

The quantum Hamiltonian of the generalised Dirac magnetic monopole is nothing else but the Bochner Laplacian acting in the sections of the corresponding line bundle, which form the space of the induced representation $\operatorname{ind}_{H}^{G}\left(\chi_{f}\right)$. Its spectrum can be computed using the Frobenius reciprocity and Kostant's multiplicity formula (see the details in [10]).

\section{Acknowledgements}

We are grateful to Ivailo Mladenov, Alex Strohmaier and especially to Alexey Bolsinov for useful discussions.

\section{References}

[1] A.V. Bolsinov and B. Jovanovic, Magnetic flows on homogeneous spaces, Comm. Math. Helv. 83 (2008), 3, 679700

[2] R. Bott, On induced representations. In The mathematical heritage of Hermann Weyl (Durham, NC, 1987), 1-13, Proc. Sympos. Pure Math. 48, Amer. Math. Soc., Providence, RI, 1988. 
[3] S. Coleman, The magnetic monopole 50 years later, In The Unity of the Fundamental Interactions (1983), A. Zichichi, editor.

[4] P.A.M. Dirac, Quantised singularities in the electromagnetic field, Proc. Roy. Soc. A 133 (1931), 60-72.

[5] D.I. Efimov, The magnetic geodesic flows on a homogeneous symplectic manifold, Siberian Math. Journal 46, no.1 (2005), 83-93.

[6] E.V. Ferapontov and A.P. Veselov, Integrable Schrödinger operators with magnetic fields: Factorization method on curved surfaces, J. Math. Phys. Vol. 42, No. 2 (2001), 590-607.

[7] M. Fierz, On the theory of particles with magnetic charge, Helv. Phys. Acta 17 (1944), 27-34.

[8] W. Fulton and J. Harris, Representation Theory: A First Course. Graduate Texts in Mathematics, 129. Springer, New York, 2004.

[9] N.E. Hurt, Geometric Quantization in Action, Mathematics and its Applications, 8. D. Reidel Publishing Company, 1983.

[10] G.M.Kemp, Algebra and geometry of Dirac's magnetic monopole, PhD Thesis, Loughborough University, June 2013.

[11] A.A. Kirillov, Geometric quantization, Dynamical systems IV, Encyclopaedia Math. Sci., 4, Springer, Berlin, 2001.

[12] A.A. Kirillov, Lectures on the Orbit Method, Graduate Studies in Mathematics, 64, American Mathematical Society, 2004.

[13] S. Kobayashi and K. Nomizu, Foundations of Differential Geometry. John Wiley and Sons, New York-London, 1963.

[14] I.M. Mladenov and V.V. Tsanov, Geometric quantisation of the MIC-Kepler problem, J. Phys. A20 (1987), no. 17, 5865-5871.

[15] S.P. Novikov and I. Schmelzer, Periodic solutions of Kirchhoff's equations for the free motion of a rigid body in a fluid and the extended theory of Lyusternik - Shnirel'man - Morse (LSM). I. Funct. Anal. Appl. Vol. 15, No. 3 (1981), 54-66.

[16] A.L. Reiman, Relativistic and Galilean-invariant classical mechanical systems, In Differential Geometry, Lie Groups and Mechanics. Zapiski Nauch. Seminarov LOMI, 37 (1973), 47-52 (in Russian).

[17] E. Schrödinger, A method of determining quantum mechanical eigenvalues and eigenfunctions, Proc. Royal Irish Acad. A 46 9-16 (1940); Further studies on solving eigenvalue problems by factorisation, ibid. 183-206 (1941).

[18] I. Tamm, Die verallgemeinerten Kugelfunktionen und die Wellenfunktionen eines Elektrons in Felde eines Magnetpoles, Zeitschrift für Physik, 71 (1931), 141-150.

[19] C. Tejero Prieto, Quantization and spectral geometry of a rigid body in a magnetic monopole field. Differential Geom. Appl. 14 (2001), no. 2, 157-179.

[20] T.T. Wu and C.N. Yang, Dirac monopole without strings: monopole harmonics, Nuclear Physics B 107 (1976), 365-380. 